\title{
A class of nonlinear adaptive observers for SIR epidemic model
}

\author{
Pierre-Alexandre Bliman ${ }^{1}$, Denis Efimov ${ }^{2}$ and Rosane Ushirobira ${ }^{2}$
}

\begin{abstract}
Mathematical epidemic models describe the spread of an infectious disease in a host population. The SIR model, which is one of the simplest, is based on the representation of interactions between three compartments in the population: the number of susceptible, infective and recovered individuals. In this note, we study the problem of state estimation for such a model, subject to seasonal variations and uncertainties in the measured incidence rate (assuming continuous measurement), and design for this purpose a class of nonlinear adaptive observers. Asymptotic stability and robustness with respect to variation rates are ensured by an appropriate choice of the observer gains as a function of the state estimate, through the use of the theory of input-to-output stability. Numerical experiments are presented to illustrate the method efficiency.
\end{abstract}

\section{INTRODUCTION}

The SIR model with vital dynamics, see e.g. [1], [2], [3], [4], is one of the most elementary compartmental model of epidemics. It describes the evolution of the relative proportions of three classes of a population of constant size, namely the susceptibles $S$, capable of contracting the disease and becoming infective; the infectives $I$, capable of transmitting the disease to susceptibles; and the recovered $R$, permanently immune after healing.

When the model parameters are constant, the evolution of such a system is quite simple: depending upon the value of the so-called basic reproduction ratio [3], [4], the system converges towards a disease-free equilibrium that always exists, or towards an asymptotically stable endemic equilibrium that appears when the previous one becomes unstable. On the contrary, when the parameters are time-varying, complicated dynamics may occur [5].

In the sequel we consider that the population is stationary, but that the conditions of transmission of the considered contagious disease depend upon time (e.g. through climate conditions or seasonal behavior of the host population). Moreover, we will consider that their knowledge is imprecise. On the other hand, the only measurement available on the system will be the host incidence, i.e. the number of new infected per unit time, an information typically accessible to the Public Health Services. Notice that this quantity is a nonlinear function of the state variable.

\footnotetext{
1 Pierre-Alexandre Bliman is with Sorbonne Université, Université Paris-Diderot SPC, CNRS, Inria, Laboratoire Jacques-Louis Lions, équipe Mamba, Paris, France and Fundação Getúlio Vargas, Rio de Janeiro, Brazil Pierre-Alexandre. Bliman@inria.fr

2 Denis Efimov and Rosane Ushirobira are with Inria, Villeneuve d'Ascq, France and CRIStAL (UMR CNRS 9189), Villeneuve d'Ascq, France Denis.Efimov, Rosane.Ushirobiradinria.fr

* This work was partially by the Government of Russian Federation (Grant 074-U01) and the Ministry of Education and Science of Russian Federation (Project 14.Z50.31.0031).
}

Our goal in this paper is to estimate at the same time the proportions of the three populations, and the value of the time-varying transmission rate. If available, such observer could be used for epidemic outbreak forecasting, or conception of early warning systems.

Notice that interval observers of the state variable alone have been proposed in [6] for the same system. Similarly to the situation presented in this reference, the epidemiological system studied here is unobservable in absence of infected hosts.

It is worth stressing that the output is a polynomial function of the state in our case, and despite the fact that the system equations are linear with respect to output and state, the observer design problem is highly nonlinear. The existing approaches cannot be applied [7], [8], so we develop a new stability and robustness analysis for the considered estimation problem, whose generalization can be an important and challenging problem for future research.

The paper is organized as follows: in Section II, the mathematical model is described, as well as the problems addressed in this work. State observers are designed in Section III and preliminary stability results are shown. In Section IV, an estimate for the time-varying transmission rate is constructed. Finally Section V contains the stability analysis for the proposed global observers. Numerical examples can be found in Section VI.

\section{PRoblem STATEMENT}

Consider a basic SIR model:

$$
\begin{aligned}
\dot{S}(t) & =\mu-\beta(t) S(t) I(t)-\mu S(t), \\
\dot{I}(t) & =\beta(t) S(t) I(t)-(\gamma+\mu) I(t), \\
\dot{R}(t) & =\gamma I(t)-\mu R(t), \\
y(t) & =\beta(t) S(t) I(t),
\end{aligned}
$$

where $S(t), I(t), R(t) \in[0,1]$ represent normalized populations of susceptible, infected and recovered groups of individuals, respectively. The quantity $y(t) \in \mathbb{R}_{+}$, seen here as the measurement output, represents the incidence rate of the disease, that is the number of new infectives per time unit. This rate is typically available from epidemiological surveillance system. The parameters $0<\mu<\gamma<+\infty$ (mortality and recovery rates, respectively) are supposed to be known, while the piecewise continuous function $\beta: \mathbb{R}_{+} \rightarrow \mathbb{R}_{+}$, which represents the infection rate, is unknown and subject to seasonal variations.

The infection induces no supplementary mortality in the population, and the latter is supposed to be stationary, so by construction $\dot{S}+\dot{I}+\dot{R} \equiv 0$. We will assume that the following 
property is initially fulfilled, and therefore conserved during the whole evolution:

$$
S(t)+I(t)+R(t)=1, \quad \forall t \geq 0 .
$$

It is straightforward to verify that the model (1) has nonnegative solutions for non-negative initial conditions (see also [6, Lemma 3.1]).

The question considered in this paper is: how to estimate the values of all populations $S(t), I(t), R(t)$ and the infection rate function $\beta(t)$, based on the measurement of $y(t)$ ? An important point is that, for relevant applications, the value of $\mu$ is quite smaller than $\gamma$, while the convergence of the observer typically needs to be guaranteed with a pace comparable to $\gamma$.

\section{OBSERVING THE POPULATION OF THE COMPARTMENTS}

Due to the structure of system (1) and the imposed assumption (2), the model can be simplified by skipping the dynamics of $R(t)$ :

$$
\begin{aligned}
\dot{S}(t) & =\mu-y(t)-\mu S(t) \\
\dot{I}(t) & =y(t)-(\gamma+\mu) I(t) \\
y(t) & =\beta(t) S(t) I(t)
\end{aligned}
$$

We introduce the following class of observers for the population variables $S(t)$ and $I(t)$ :

$$
\begin{aligned}
\dot{\widehat{S}}(t) & =\mu-y(t)-\mu \widehat{S}(t)+\ell_{1}(y(t)-\widehat{y}(t)) \\
\dot{\hat{I}}(t) & =y(t)-(\gamma+\mu) \widehat{I}(t)+\ell_{2}(y(t)-\widehat{y}(t)), \\
\widehat{y}(t) & =\widehat{\beta}(t) \widehat{S}(t) \widehat{I}(t)
\end{aligned}
$$

where $\widehat{S}(t), \widehat{I}(t), \widehat{y}(t), \widehat{\beta}(t) \in \mathbb{R}$ are estimates of $S(t), I(t), y(t)$ and $\beta(t)$, respectively. The quantities $\ell_{1}, \ell_{2} \in \mathbb{R}$ are observer gains to be adjusted. The equation for adaptation of $\widehat{\beta}(t)$ will be given later.

As it can be concluded from equations (4), the observer has the structure of the model (3) with an output injection, thus being rather standard. However, the term $\widehat{y}(t)$, used in the output injection, contains a cubic term in the estimating variables, which makes the stability analysis of this observer a complicated problem. The following result is similar to [6, Lemma 3.2], and can be proven by using results in [9], [10].

\section{Lemma 1: Let}

$$
\ell_{1} \geq 1, \ell_{2} \geq-1
$$

then

$$
\widehat{S}(t) \geq 0, \widehat{I}(t) \geq 0 \quad \forall t>0
$$

provided that $\widehat{S}(0) \geq 0, \widehat{I}(0) \geq 0$.

The non-linearity of the observer (4) is evident considering the dynamics of estimation errors:

$$
e_{S}(t)=S(t)-\widehat{S}(t), \quad e_{I}(t)=I(t)-\widehat{I}(t),
$$

which takes the form:

$$
\begin{aligned}
\dot{e}_{S}(t)= & -\mu e_{S}(t)-\ell_{1}(y(t)-\widehat{y}(t)) \\
= & -\left(\mu+\ell_{1} \beta(t) \widehat{I}(t)\right) e_{S}(t)-\ell_{1}\left[\beta(t) \widehat{S}(t) e_{I}(t)\right. \\
& \left.+\beta(t) e_{S}(t) e_{I}(t)+(\beta(t)-\widehat{\beta}(t)) \widehat{S}(t) \widehat{I}(t)\right], \quad(6 \mathrm{a}) \\
\dot{e}_{I}(t)= & -(\gamma+\mu) e_{I}(t)-\ell_{2}(y(t)-\widehat{y}(t)) \\
= & -\left(\gamma+\mu+\ell_{2} \beta(t) \widehat{S}(t)\right) e_{I}(t)-\ell_{2}\left[\beta(t) \widehat{I}(t) e_{S}(t)\right. \\
& \left.+\beta(t) e_{S}(t) e_{I}(t)+(\beta(t)-\widehat{\beta}(t)) \widehat{S}(t) \widehat{I}(t)\right],
\end{aligned}
$$

where the equations were developed with respect to the observer variables $\widehat{S}(t), \widehat{I}(t)$ for ease of further analysis. The system (6) can be interpreted as a time-varying system (through the terms $\beta(t), \widehat{I}(t), \widehat{S}(t)$ and their products) with the external input $(\beta(t)-\widehat{\beta}(t)) \widehat{S}(t) \widehat{I}(t)$.

Let us show that under mild conditions the system (3), (4) is uniformly input-to-output stable (IOS) [11], [12] with respect to this input and the estimation errors $e_{S}$ and $e_{I}$ as the outputs (to show input-to-state stability of the system, an additional analysis of the dynamics of (3) would have to be performed). For this, introduce the following Lyapunov function candidate for (6):

$$
W\left(e_{S}, e_{I}\right)=\left|e_{S}\right|+\frac{\ell_{1}}{\ell_{2}}\left|e_{I}\right|,
$$

which is positive definite for

$$
\ell_{1} \geq 1, \ell_{2}>0
$$

satisfying (5).

Lemma 2: Assume that the condition (8) is verified and that

$$
\left|e_{S}(t)\right| \leq \lambda,\left|e_{I}(t)\right| \leq \lambda, \quad \forall t \in[0, T)
$$

for some $0<\lambda \leq 1$ and $T>0$. Then for $\varepsilon \in(0, \mu)$

$$
W(t) \leq e^{-\varepsilon t} W(0)+2 \frac{\ell_{1}}{\varepsilon} \sup _{\tau \in[0, t]}|\beta(\tau)-\widehat{\beta}(\tau)||\widehat{S}(\tau) \widehat{I}(\tau)|
$$

for all $t \in[0, T)$ provided that

$$
2 \ell_{1} \beta \leq \frac{\mu-\varepsilon}{\lambda}+\frac{\ell_{1}}{\ell_{2}} \frac{\gamma+\mu-\varepsilon}{\lambda} .
$$

The proof of this lemma is based on Lemma 1 and on the following estimate for the Lyapunov function $W$ :

$$
\begin{aligned}
\dot{W} \leq & -\varepsilon W-(\mu-\varepsilon)\left|e_{S}\right|-\frac{\ell_{1}}{\ell_{2}}(\gamma+\mu-\varepsilon)\left|e_{I}\right| \\
& +2 \ell_{1} \beta\left|e_{I}\right|\left|e_{S}\right|+2 \ell_{1}|\beta-\widehat{\beta} \| \widehat{S I}|
\end{aligned}
$$

for some $0<\varepsilon<\mu$.

Thus, under the conditions (8), (9) and (10), the system (3), (4) possesses a kind of uniform IOS property with respect to the input $(\beta(t)-\widehat{\beta}(t)) \widehat{S}(t) \widehat{I}(t)$ and the output $\left[e_{I} e_{S}\right]^{T}$, and $W$ defined in (7) is its IOS Lyapunov function. Note that the restriction 9 for $\lambda \leq 1$ does not imply a local result since $S(t), I(t) \in[0,1]$ for all $t \geq 0$, then the estimation errors may stay in the interval $[-1,1]$. 


\section{EStimating THE TRANSMission RATE $\beta$}

We now complete the observer equation (4) by defining an observer for $\beta(t)$ as follows:

$$
\dot{\widehat{\beta}}(t)=\rho(y(t)-\widehat{y}(t)) \operatorname{sign}(\widehat{S}(t) \widehat{I}(t)),
$$

where $\rho>0$ is a tuning parameter, $\widehat{\beta}(0) \geq 0$. Since $\operatorname{sign}(\widehat{S}(t) \widehat{I}(t))=1$ under the condition $\sqrt{5})$, then the sign can be skipped in the formula above.

Notice that

$$
\begin{aligned}
y(t)-\widehat{y}(t)= & \beta(t) S(t) I(t)-\widehat{\beta}(t)) \widehat{S}(t) \widehat{I}(t) \\
= & (\beta(t)-\widehat{\beta}(t)) \widehat{S}(t) \widehat{I}(t) \\
& +\beta(t)\left[e_{S}(t) e_{I}(t)+\widehat{I}(t) e_{S}(t)+\widehat{S}(t) e_{I}(t)\right],
\end{aligned}
$$

and (12) can then be rewritten as follows:

$$
\begin{aligned}
\dot{\hat{\beta}}(t)= & \rho(\beta(t)-\widehat{\beta}(t))|\widehat{S}(t) \widehat{I}(t)| \\
& +\rho \beta(t)\left[e_{S}(t) e_{I}(t)+\widehat{I}(t) e_{S}(t)\right. \\
& \left.+\widehat{S}(t) e_{I}(t)\right] \operatorname{sign}(\widehat{S}(t) \widehat{I}(t)),
\end{aligned}
$$

representing the observer 12 as a linear time-varying system in terms of the parameter estimation error $e_{\beta}=\beta-\widehat{\beta}$ and an additive input proportional to the population estimation errors $e_{S}, e_{I}$ and their product.

Let us now show that the complete system (3), (4), (12) admits a uniform IOS property with respect to the output $e_{\beta}$ and the input $\beta\left(e_{S} e_{I}+\widehat{I} e_{S}+\widehat{S} e_{I}\right)+\dot{\beta}$. To this end consider the following Lyapunov function:

$$
U\left(e_{\beta}\right)=\left|e_{\beta}\right|
$$

Lemma 3: Assume that

$$
|\widehat{S}(t) \widehat{I}(t)| \geq \eta \quad \forall t \in[0, T)
$$

for some $\eta>0$ and $T>0$, then for all $t \in[0, T)$

$$
\begin{aligned}
U(t) \leq & e^{-\rho \eta t} U(0)+\eta^{-1} \sup _{\tau \in[0, t]}\left\{\beta(\tau) \mid e_{S}(\tau) e_{I}(\tau)\right. \\
& \left.+\widehat{I}(\tau) e_{S}(\tau)+\widehat{S}(\tau) e_{I}(\tau)\left|+\rho^{-1}\right| \dot{\beta}(\tau) \mid\right\}
\end{aligned}
$$

The proof of this lemma uses the following estimate on the derivative of $U$ :

$$
\dot{U} \leq-\rho\left|e_{\beta}\right||\widehat{S I}|+\rho \beta\left|e_{S} e_{I}+\widehat{I} e_{S}+\widehat{S} e_{I}\right|+|\dot{\beta}(t)| .
$$

The restriction (14) is a kind of persistence of excitation condition, which is a usual requirement for estimation of parameters in adaptive control and identification [8]. It is typically verified on finite intervals of time, during the epidemic bursts.

\section{STABILITY ANALYSIS AND MAIN RESUlT}

Under some mild conditions for the model (3), we have established that the state observer (4) is IOS from the input proportional to the parameter estimation error $e_{\beta}$ to the output represented by the state estimation errors $\left[\begin{array}{ll}e_{I} & e_{S}\end{array}\right]^{T}$, while the parameter observer (12) admits a similar kind of property from the input proportional to $\left[e_{I} e_{S}\right]^{T}$ and the output $e_{\beta}$ with an external input $\dot{\beta}$. Thus, both observers form a feedback interconnection, which is stable under a properly established small-gain condition [13].

Consider the following Lyapunov function for the complete system (3), (4), (12):

$$
V\left(e_{S}, e_{I}, e_{\beta}\right)=W\left(e_{S}, e_{I}\right)+b U\left(e_{\beta}\right)=\left|e_{S}\right|+\frac{\ell_{1}}{\ell_{2}}\left|e_{I}\right|+b\left|e_{\beta}\right|,
$$

where $b>0$ is a constant parameter defined later. Under $(8)$ and following (11) and (15), the derivative of this Lyapunov function admits an upper estimate:

$$
\begin{aligned}
\dot{V} \leq & -\mu\left|e_{S}\right|-\frac{\ell_{1}}{\ell_{2}}(\gamma+\mu)\left|e_{I}\right|+2 \ell_{1} \beta\left|e_{I}\right|\left|e_{S}\right| \\
& +\left(2 \ell_{1}-b \rho\right)\left|e_{\beta}\right| \widehat{S I}+b \rho \beta\left|e_{S} e_{I}+\widehat{I} e_{S}+\widehat{S} e_{I}\right|+b|\dot{\beta}| \\
\leq & -(\mu-b \rho \beta \widehat{I})\left|e_{S}\right|-\left\{\frac{\ell_{1}}{\ell_{2}}(\gamma+\mu)-b \rho \beta \widehat{S}\right\}\left|e_{I}\right| \\
& +\beta\left(2 \ell_{1}+b \rho\right)\left|e_{I}\right|\left|e_{S}\right|+\left(2 \ell_{1}-b \rho\right)\left|e_{\beta}\right| \widehat{S I}+b|\dot{\beta}| .
\end{aligned}
$$

Taking into account the result of Lemma 1, the condition (9) and imposing $S(t), I(t) \in(0,1)$ we obtain that

$$
0<\widehat{S}(t) \leq 1+\lambda, 0<\widehat{I}(t) \leq 1+\lambda
$$

for all $t \in[0, T)$. Then for $b \rho=(2+\alpha) \ell_{1}$, where $\alpha>0$ is a design parameter, we get:

$$
\begin{aligned}
\dot{V} \leq & -\left(\mu-(2+\alpha) \ell_{1} \beta \widehat{I}\right)\left|e_{S}\right| \\
& -\left\{\ell_{1} \ell_{2}^{-1}(\gamma+\mu)-(2+\alpha) \ell_{1} \beta \widehat{S}\right\}\left|e_{I}\right| \\
& +(4+\alpha) \beta \ell_{1}\left|e_{I}\right|\left|e_{S}\right|-\alpha \ell_{1}\left|e_{\beta}\right| \widehat{S I}+b|\dot{\beta}| \\
= & -\varepsilon W-\alpha \ell_{1}\left|e_{\beta}\right| \widehat{S} \widehat{I}+b|\dot{\beta}|+g\left(e_{I}, e_{S}\right), \\
g\left(e_{I}, e_{S}\right)= & -\left(\mu-\varepsilon-(2+\alpha) \ell_{1} \beta \widehat{I}\right)\left|e_{S}\right| \\
& -\ell_{1}\left\{\ell_{2}^{-1}(\gamma+\mu-\varepsilon)\right. \\
& -(2+\alpha) \beta \widehat{S}\}\left|e_{I}\right|+(4+\alpha) \beta \ell_{1}\left|e_{I}\right|\left|e_{S}\right|
\end{aligned}
$$

with $\varepsilon \in(0, \mu)$. Now, we would like to guarantee the following series of inequalities, by an adequate choice of $\ell_{1}$ and $\ell_{2}$ :

$$
\begin{gathered}
\mu-\varepsilon-(2+\alpha) \ell_{1} \beta \widehat{I}>0, \\
\ell_{2}^{-1}(\gamma+\mu-\varepsilon)-(2+\alpha) \beta \widehat{S}>0, \\
g\left(e_{I}, e_{S}\right) \leq 0
\end{gathered}
$$

in order to conclude

$$
\dot{V} \leq-\varepsilon W-\alpha \ell_{1}\left|e_{\beta}\right| \widehat{S} \widehat{I}+b|\dot{\beta}|
$$

Under (9) this series of inequalities has a solution for $\ell_{1}$ and $\ell_{2}$ if:

$$
\begin{gathered}
\frac{\gamma+\mu-\varepsilon}{\beta((4+\alpha) \lambda+(2+\alpha)(\widehat{S}+\widehat{I}))}<\ell_{2}<\frac{\gamma+\mu-\varepsilon}{(2+\alpha) \beta(\widehat{S}+\widehat{I})}, \\
\frac{\mu-\varepsilon}{(2+\alpha) \beta \widehat{I}}>\ell_{1} \geq 1,
\end{gathered}
$$

where all bounds are either functions of known parameters (i.e. $\mu, \gamma$ ) and tuning gains (i.e. $\varepsilon, \lambda$ and $\alpha$ ) or measured variables $\widehat{S}(t)$ and $\widehat{I}(t)$, and the only uncertain variable is $\beta(t)$.

We are now in position to state our main result. 
Theorem 1: Assume that

$$
\beta(t) \in\left[\beta_{\min }, \beta_{\max }\right], \quad \forall t \in[0, T)
$$

for some known $\beta_{\min }, \beta_{\max } \in \mathbb{R}_{+}$, where

$$
\beta_{\min }<\beta_{\max }<\frac{3}{2} \beta_{\min } .
$$

Let $\varepsilon, \kappa, \alpha$ be positive constants such that

$$
\varepsilon<\mu, \quad \kappa<0.25, \quad \beta_{\max }<\beta_{\min }\left(1+\frac{4+\alpha}{8+4 \alpha}\right) .
$$

Choose the gains $\ell_{1}, \ell_{2}$ such that $\ell_{2} \leq \ell_{1}$ for any $t$, and

$$
\begin{gathered}
\frac{\gamma+\mu-\varepsilon}{\beta_{\min }(4+\alpha+(2+\alpha)(\widehat{S}(t)+\widehat{I}(t)))}<\ell_{2} \\
<\frac{\gamma+\mu-\varepsilon}{\beta_{\max }(2+\alpha)(\widehat{S}(t)+\widehat{I}(t))}, \\
1 \leq \ell_{1}<\frac{\mu-\varepsilon}{\beta_{\max }(2+\alpha) \widehat{I}(t)},
\end{gathered}
$$

and the initial conditions such that

$$
e_{S}(0) \leq \kappa, e_{I}(0) \leq \kappa \frac{\ell_{2}}{\ell_{1}}, e_{\beta}(0) \leq \frac{\beta_{\max }-\beta_{\min }}{2} .
$$

Then on any interval $[0, T]$ on which $(14)$ holds, the condition (9) is satisfied with $\lambda=1$, and

$$
\begin{array}{r}
V(t) \leq \mathrm{e}^{-\min \left\{\varepsilon, \frac{\eta \alpha}{b}\right\} t} V(0)+\max \left\{\frac{b}{\varepsilon}, \frac{1}{\eta \alpha}\right\} \sup _{s \in[0, t)}|\dot{\beta}(s)|, \\
\forall t \in[0, T),
\end{array}
$$

for any constants $b$ and $\eta$ that fulfill

$$
b \leq 2 \frac{\kappa}{\beta_{\max }-\beta_{\min }},
$$

$$
\sup _{t \in[0, T)}|\dot{\beta}(t)| \leq \min \left\{\varepsilon \frac{\beta_{\max }-\beta_{\min }}{2}, \eta \alpha \kappa\right\} \text {. }
$$

Note that due to imposed restrictions on the initial conditions $e_{S}(0), e_{I}(0)$ and $e_{\beta}(0)$, the obtained stability results have a local nature.

Proof: It is straightforward to verify that the interval

$$
\left(\frac{\gamma+\mu-\varepsilon}{\beta_{\min }(4+\alpha+(2+\alpha)(\widehat{S}+\widehat{I}))}, \frac{\gamma+\mu-\varepsilon}{\beta_{\max }(2+\alpha)(\widehat{S}+\widehat{I})}\right)
$$

is not empty under the introduced restriction 20 , and the inequalities (18) with $\lambda=1$ follow from 21. Since $\kappa<0.25$ and $\ell_{2} \leq \ell_{1}$, the condition (9) with $\lambda=1$ is true at $t=0$. Assume by contradiction that the estimate (17) is satisfied for $t \in\left[0, t^{\prime}\right)$ with some $t^{\prime}>0$ when the condition 9$]$ is the first time violated. If the restriction (14) is valid for some $\eta>0$, then 17 can be rewritten as follows:

$$
\dot{V} \leq-\rho V+b \sup _{s \in[0, t)}|\dot{\beta}(s)|, \rho=\min \left\{\varepsilon, \frac{\eta \alpha \ell_{1}}{b}\right\}
$$

for all $t \in\left[0, t^{\prime}\right)$, and thus:

$$
V(t) \leq e^{-\rho t} V(0)+\frac{b}{\rho} \sup _{s \in[0, t)}|\dot{\beta}(s)|
$$

for all $t \in\left[0, t^{\prime}\right)$. Since $V(0) \leq 2 \kappa+b \frac{\beta_{\max }-\beta_{\min }}{2}$ and

$$
V(t) \leq 2 \kappa+b \frac{\beta_{\max }-\beta_{\min }}{2}+\frac{b}{\rho} \sup _{s \in[0, t)}|\dot{\beta}(s)|
$$

the conditions 23 imply that

$$
b \frac{\beta_{\max }-\beta_{\min }}{2} \leq \kappa, \frac{b}{\rho} \sup _{t \in[0, T)}|\dot{\beta}(t)| \leq \kappa,
$$

which results in

$$
V(t) \leq 4 \kappa<1 \quad t \in\left[0, t^{\prime}\right)
$$

that contradicts the definition of $t^{\prime}$. Therefore, the condition (9) with $\lambda=1$ and 17 are true for all $t \in[0, T)$.

Remark 1: It is straightforward to check that the condition 19] is equivalent to the existence of $\alpha>0$ fulfilling the last inequality of (20).

Remark 2: Under the hypotheses of Theorem 1, if in addition $\dot{\beta}(t)=0$ and all the other properties are satisfied for all $t \geq 0$, then

$$
\lim _{t \rightarrow+\infty} e_{S}(t)=\lim _{t \rightarrow+\infty} e_{I}(t)=\lim _{t \rightarrow+\infty} e_{\beta}(t)=0 .
$$

According to Theorem 1, in order to implement the observer (4), (12), the gains $\ell_{1}, \ell_{2}$ and $\rho$ can be selected as follows:

$$
\begin{gathered}
\ell_{1}=\max \left\{1, \frac{\mu-\varepsilon}{(2+\alpha) \beta_{\max } \widehat{I}}\right\}, \\
\ell_{2}=\min \left\{1, \frac{\gamma+\mu-\varepsilon}{\beta_{\max }(2+\alpha)(\widehat{S}+\widehat{I})}\right\}, \\
\rho=\ell_{1} \frac{2+\alpha}{2 \kappa}\left(\beta_{\max }-\beta_{\min }\right),
\end{gathered}
$$

where $\varepsilon \in(0, \mu), \kappa \in(0,0.25)$ and $\alpha>0$ are free tuning parameters, $\kappa$ is related with initial estimation error. From the inequality

$$
\widehat{I}(t) \leq \frac{\mu-\varepsilon}{\beta_{\max }(2+\alpha)}
$$

the validity of conditions of Theorem 1 can be monitored (it is suitable for initial phase of an epidemic). Since the value of $\ell_{1}$ is growing for small values of $\widehat{I}$, then an additional saturation of this gain is desirable during implementation.

Remark 3: Adapting the considerations in [14], one may show that the condition (14) can be replaced with the following relaxed restriction:

$$
\int_{0}^{t}|\widehat{S}(s) \widehat{I}(s)| d s \geq \eta t, \quad \forall t \in[0, T)
$$

for some $T>0$ and $\eta>0$, at the price of more complex mathematical statements and estimates on upper bounds of trajectories and convergence rates. The proof is omitted here for the sake of space.

Remark 4: The designed observer guarantees the estimation of the system states $S, I$ and the time-varying parameter $\beta$ with a static error proportional to $\dot{\beta}$. To improve its 


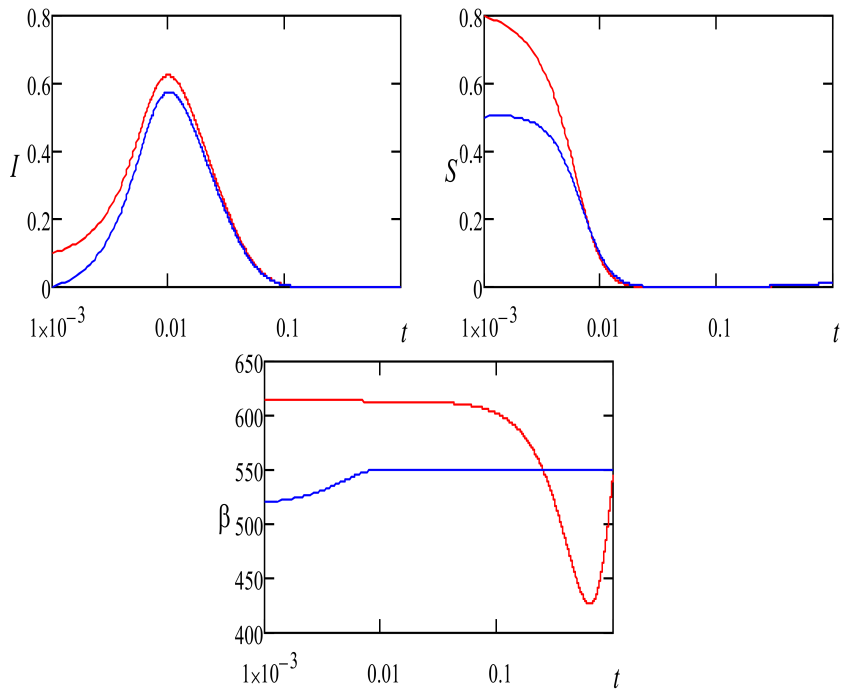

Fig. 1. The results of estimation for 12

accuracy, a sliding mode term can be introduced in (12) through the equation:

$$
\dot{\widehat{\beta}}(t)=\left[\rho(y(t)-\widehat{y}(t))+\rho^{\prime} \operatorname{sign}(y(t)-\widehat{y}(t))\right] \operatorname{sign}(\widehat{S}(t) \widehat{I}(t)),
$$

where $\rho^{\prime}>0$ is another tuning gain admitting the restriction:

$$
\rho^{\prime} \geq b \sup _{t \in[0, T)}|\dot{\beta}(t)|
$$

The corresponding proof is omitted here for the sake of space.

\section{NUMERICAL EXPERIMENTS}

Select

$$
\mu=\frac{1}{70} \text { year }^{-1}, \gamma=\frac{365}{7} \text { year }^{-1}, \beta_{\min }=416, \beta_{\max }=624,
$$

and for simulation

$$
\beta(t)=520(1+0.18 \cos (5 t)) .
$$

Take $\ell_{1}, \ell_{2}, \rho$ as in 24 with $\varepsilon=0.1 \mu, \kappa=0.1$ and $\alpha=$ 0.1 (the values of $\ell_{1}$ and $\rho$ will be saturated by 100 during simulation), and

$S(0)=0.6, I(0)=10^{-6}, \widehat{S}(0)=0.5, \widehat{I}(0)=10^{-7}, \widehat{\beta}(0)=520$,

then the conditions of Theorem 1 are satisfied. The results of the estimation for the observer (4), (12) and $T=1$ year are shown in Fig. 11 and for the observer (4), (25) in Fig. 2) (the blue lines represent the estimates generated by the observers, while the red lines correspond to outputs of the model (3p). As we can conclude from these results, the estimations of $S$ and $I$ are always obtained faster than in 1 month, while the estimation of $\beta$ is well provided by 25.

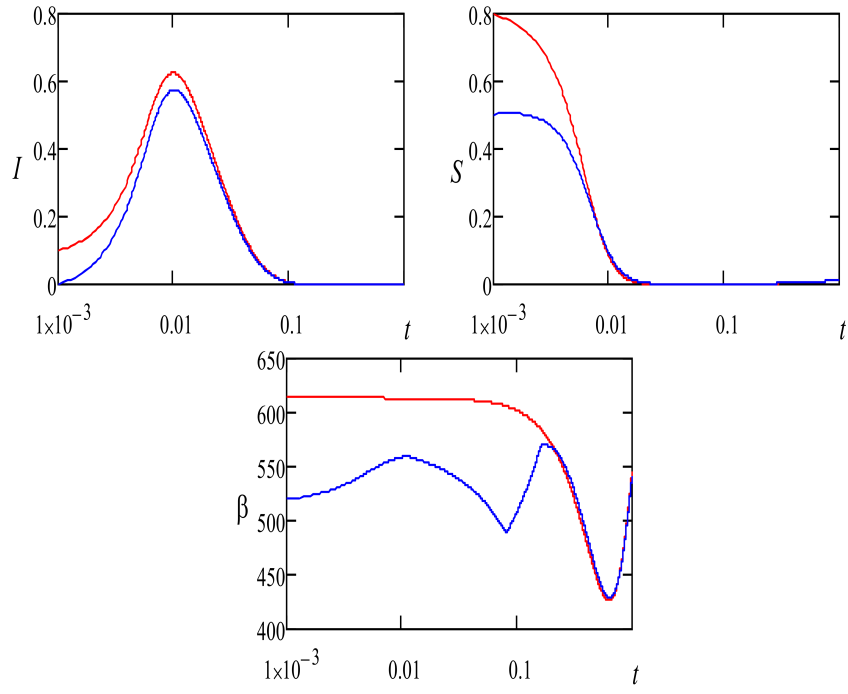

Fig. 2. The results of estimation for 25

\section{CONCLUSION}

The problem of estimating the states $S(t), I(t)$ and the time-varying parameter $\beta(t)$ has been considered for a SIR model (3) using the incidence rate $y(t)=\beta(t) I(t) S(t)$ as the (nonlinear) measured signal, where the transmission rate $\beta(t)$ is uncertain. A nonlinear adaptive observer has been designed ensuring the estimation with a bounded error proportional to the variation speed $\dot{\beta}$ of the parameter $\beta$. The stability and robustness properties are established analytically using the theory of input-to-output stability and the small-gain approach. The efficiency of the obtained solution is demonstrated in numerical experiments. As a direction of future research the development of a discrete measurement-based observer can be indicated. Another interesting feature for further analysis is robustness with respect to the measurement noise of this nonlinear adaptive observer.

\section{REFERENCES}

[1] N. T. Bailey et al., The mathematical theory of infectious diseases and its applications. Charles Griffin \& Company Ltd, 5a Crendon Street, High Wycombe, Bucks HP13 6LE., 1975.

[2] R. M. Anderson, R. M. May, and B. Anderson, Infectious diseases of humans: dynamics and control. Wiley Online Library, 1992, vol. 28.

[3] V. Capasso, Mathematical structures of epidemic systems. Springer, 1993, vol. 88.

[4] M. J. Keeling and P. Rohani, Modeling infectious diseases in humans and animals. Princeton University Press, 2008.

[5] Y. A. Kuznetsov and C. Piccardi, "Bifurcation analysis of periodic SEIR and SIR epidemic models," Journal of mathematical biology, vol. 32, no. 2, pp. 109-121, 1994.

[6] P.-A. Bliman and B. D. Barros, "Interval observers for SIR epidemic models subject to uncertain seasonality," in Positive Systems, ser. Lecture Notes in Control and Information Sciences, Filippo Cacace et al., Ed., vol. 471. Springer, 2017.

[7] T. Fossen and H. Nijmeijer, New Directions in Nonlinear Observer Design. Springer, 1999.

[8] G. Besançon, Ed., Nonlinear Observers and Applications, ser. Lecture Notes in Control and Information Sciences. Springer, 2007, vol. 363.

[9] H. Smith, Monotone Dynamical Systems: An Introduction to the Theory of Competitive and Cooperative Systems, ser. Surveys and Monographs. Providence: AMS, 1995, vol. 41. 
[10] W. Haddad and V. Chellaboina, "Stability theory for nonnegative and compartmental dynamical systems with time delay," Syst. Control Letters, vol. 51, pp. 355-361, 2004.

[11] E. Sontag, "On the input-to-state stability property," European $J$. Control, vol. 1, pp. 24-36, 1995.

[12] S. Dashkovskiy, D. Efimov, and E. Sontag, "Input to state stability and allied system properties," Automation and Remote Control, vol. 72, no. 8, pp. 1579-1614, 2011.

[13] S. Dashkovskiy, B. S. Rüffer, and F. R. Wirth, "An ISS small gain theorem for general networks," Mathematics of Control, Signals, and Systems, vol. 19, no. 2, pp. 93-122, 2007.

[14] D. Efimov and A. Fradkov, "Design of impulsive adaptive observers for improvement of persistency of excitation," Int. J. Adaptive Control and Signal Processing, vol. 29, no. 6, pp. 765-782, 2015. 